\title{
Optimizing Thermodynamic Cycles with Two Finite-Sized Reservoirs
}

\author{
Hong Yuan, ${ }^{1}$ Yu-Han Ma, ${ }^{1, *}$ and C. P. Sun ${ }^{1,2, \dagger}$ \\ ${ }^{1}$ Graduate School of China Academy of Engineering Physics, \\ No. 10 Xibeiwang East Road, Haidian District, Beijing, 100193, China \\ ${ }^{2}$ Beijing Computational Science Research Center, Beijing 100193, China
}

\begin{abstract}
We study the non-equilibrium thermodynamics of a heat engine operating between two finite-sized reservoirs with well-defined temperatures. Within the linear response regime, it is found that the uniform temperature of the two reservoirs at final time $\tau$ is bounded from below by the entropy production $\sigma_{\min } \propto 1 / \tau$. We discover a general power-efficiency trade-off depending on the ratio of heat capacities $(\gamma)$ of the reservoirs for the engine. And a universal efficiency at maximum average power of the engine for arbitrary $\gamma$ is obtained. For practical purposes, the operation protocol of an ideal gas heat engine to achieve the optimal performance associated with $\sigma_{\min }$ is demonstrated. Our findings can be used to develop an general optimization scenario for thermodynamic cycles with finite-sized reservoirs in real-world circumstances.
\end{abstract}

Introduction.-The thermodynamic constraints exist in all kinds of energy-conversion machines. Among these constraints, Carnot efficiency serves as the upper bound for efficiency of heat engines. Such a bound is only achieved by reversible thermodynamic cycles under quasi-static limit [1], and is therefore not tight for practical heat engines with finite cycle time. Considering the restriction of operation time, abundant tighter thermodynamic constraints were obtained for finite-time thermodynamic cycles [2-6]. For example, efficiency at maximum power (EMP) [7-17], trade-off relation between power and efficiency [18-23], and thermodynamic uncertainty relation (TUR) $[24,25]$. In particular, the power-efficiency trade-off determines the feasible operation regime for finite-time heat engines and has attracted considerable attention.

Recently, to deal with another practicality that the heat is basically stored by a finite amount of material with finite heat capacity, the finiteness of the reservoir size is also taken into account as a physical restriction on thermodynamic cycles [26-33]. This issue is crucial for responding to the increasingly severe energy crisis with limited material resources. And the efficiency at maximum work (EMW) [26, 28, 33, 34] and efficiency at maximum average power (EMAP) [27, 29, 30, 33] were proposed as typical thermodynamic constraints in this case.

As two fundamental restrictions in energy conversion processes, the finiteness of operation time and reservoir size usually coexist in real-world circumstances. Hence, a more practical question naturally arises: Is there a power-efficiency trade-off associated with finitesized reservoirs? In this Letter, we address this question by studying the finite-time performance of a linear irreversible heat engine operating between two finite-sized reservoirs. We discover a general trade-off relation between power and efficiency. And a universal EMAP is

\footnotetext{
*yhma@gscaep.ac.cn

$\dagger$ suncp@gscaep.ac.cn
}
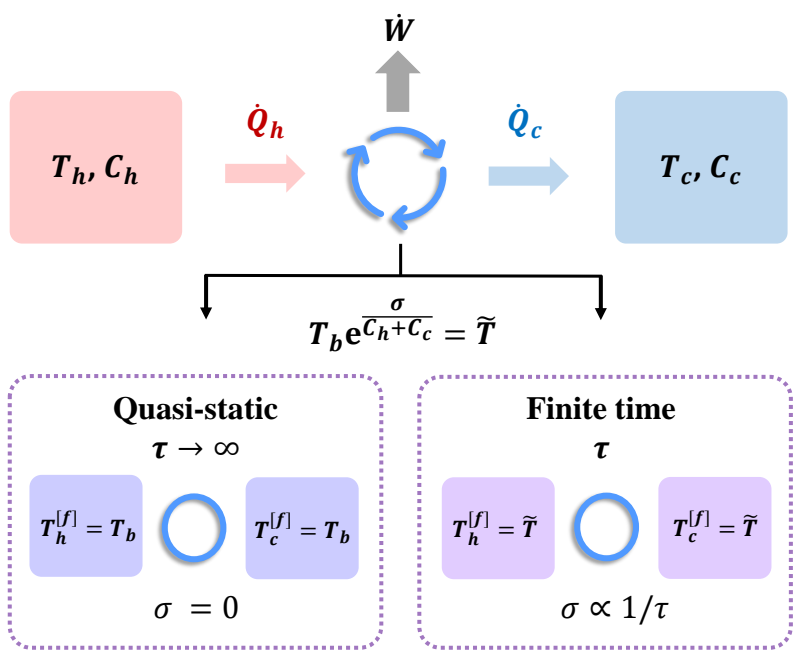

Figure 1. Demonstration of a heat engine operating between two finite-sized heat reservoirs. The heat engine operates between a finite-sized hot (cold) reservoir with initial temperature $T_{h}^{[i]}\left(T_{c}^{[i]}\right)$. The heat engine stops working when the two reservoirs reach a the final uniform temperature $T_{c}^{[f]}=T_{h}^{[f]} \equiv \tilde{T} . C_{c}\left(C_{h}\right)$ denotes the heat capacity of the cold (hot) reservoir. The increase in entropy production $\sigma$ will higher the final temperature $\tilde{T}$ [Eq. (2)].

obtained. Furthermore, we find the optimal operation of the engine to achieve the boundary of the trade-off.

The minimum entropy production and the uniform temperature.- As illustrated in Fig. 1, we consider a linear irreversible heat engine operating between a hot reservoir with initial temperature $T_{h}^{[i]}$ and a cold reservoir with initial temperature $T_{c}^{[i]}$. Both of these two reservoirs are of finite size with the heat capacity $C_{h}$ and $C_{c}$, respectively. As follows, we focus on the case of constant heat capacity $C_{h(c)}$. From the initial time $t=0$, the engine converts the heat to work consecutively through a control parameter $\lambda$ until the two reservoirs finally reach the thermal equilibrium state at $t=\tau$ with a uniform temperature $T_{c}^{[f]}=T_{h}^{[f]} \equiv \tilde{T}$. Here, we stress that the 
heat capacity of at least one reservoir needs to be finite, otherwise the temperature of the two reservoirs will always maintain their initial values instead of reaching the same within finite time. In the following, we adopt the assumptions used in the Refs. [29, 30, 33]: (i) both of the two reservoirs relax rapidly such that they are always in the quasi-equilibrium states with time-dependent temperatures $T_{h}(t)$ and $T_{c}(t)$; (ii) the total operation time $\tau$ (macro time scale) is much larger than the cycle time $\tau_{\mathrm{c}}$ (micro time scale, treat as a unit of time hereafter), and hence the engine undergoes sufficiently many cycles, namely, $M \equiv \tau / \tau_{c} \gg 1$, before it stops operating.

The entropy production rate reads $\dot{\sigma}=-\dot{Q}_{h} / T_{h}+$ $\dot{Q}_{c} / T_{c}$, where $\dot{Q}_{h}=-C_{h} \dot{T}_{h}$ represents the heat absorption from the hot reservoir to the engine of a cycle, and $\dot{Q}_{c}=C_{c} \dot{T}_{c}$ is the heat release from the engine to the cold reservoir of a cycle. As a result, the total entropy production $\sigma(\tau) \equiv \int_{0}^{\tau} \dot{\sigma} d t$ is

$$
\sigma(\tau)=C_{c} \ln \frac{\tilde{T}}{T_{c}^{[i]}}+C_{h} \ln \frac{\tilde{T}}{T_{h}^{[i]}}
$$

The uniform temperature $\tilde{T}$ is thus determined by the entropy production as

$$
\tilde{T}=\tilde{T}(\sigma)=\left[T_{h}^{[i]}\right]^{\frac{1}{\gamma+1}}\left[T_{c}^{[i]}\right]^{\frac{\gamma}{\gamma+1}} \exp \left[\frac{\sigma}{C_{h}+C_{c}}\right]
$$

namely the uniform temperature rises as the entropy production increases. Here the heat capacity ratio $\gamma \equiv$ $C_{c} / C_{h}$ quantifies the asymmetry in size of the reservoirs. $T_{b} \equiv \tilde{T}(\sigma=0)$ is the final temperature in the reversible case with no entropy production. The reversible case is discussed in the Supplementary Materials (SM) [35].

Then, we exploit the linear irreversible thermodynamics to obtain $\sigma(\tau)$ as well as $\tilde{T}$ explicitly in the finite-time regime. Under the tight-coupling condition $q \equiv L_{21} / \sqrt{L_{11} L_{22}}=1$, the entropy production rate reads $[29,30]$

$$
\dot{\sigma}=\frac{\dot{Q}_{h}^{2}}{L_{22}}=\frac{C_{h}^{2} \dot{T}_{h}^{2}}{L_{22}}
$$

where $L_{i j}(i, j=1,2)$ is the Onsager coefficient, and $L_{22}$ corresponds to the thermal conductivity [36-38]. The adopted tight-coupling condition can be practical realized, e.g., by a finite-time ideal gas Carnot engine [36].

The Cauchy-Schwarz (C-S) inequality

$$
\left[\int_{0}^{\tau}(\sqrt{\dot{\sigma}})^{2} d t\right]\left(\int_{0}^{\tau} d t\right) \geq\left(\int_{0}^{\tau} \sqrt{\dot{\sigma}} d t\right)^{2}
$$

implies that the entropy production $\sigma(\tau)=\int_{0}^{\tau} \dot{\sigma} d t=$ $\int_{0}^{\tau}(\sqrt{\dot{\sigma}})^{2} d t$ has a lower bound, namely, [35]

$$
\sigma(\tau) \geq \frac{\Sigma_{\min }}{\tau} \equiv \sigma_{\min }
$$

In this inequality, only the first order of $\tau^{-1}$ is kept in the long-time regime [16], and the equal sign is saturated with constant entropy production rate, i.e., $\dot{\sigma}=\Sigma_{\min } / \tau^{2}$ $\left(\dot{Q}_{h}=\sqrt{L_{22} \Sigma_{\min }} / \tau\right)$. The minimum dissipation coefficient $\Sigma_{\min } \equiv\left(\int_{T_{h}^{[i]}}^{T_{b}} C_{h} d T_{h} / \sqrt{L_{22}}\right)^{2}$, characterizing how irreversible entropy production increases away from the reversible regime, is a $\tau$-independent dissipation coefficient. Generally, $\Sigma_{\min }$ depends on the specific form of $L_{22}$ and relates to the thermodynamic length [38-41]. In the simplest case with constant $L_{22}, \Sigma_{\min }=C_{h}^{2}\left[T_{h}^{[i]}-T_{b}\right]^{2} / L_{22}$. The typical $1 / \tau$-scaling of irreversibility shown in Eq. (5) has also been discovered in the finite-time isothermal processes [13, 23, 42-44].

We remark here that although the minimum entropy production $\sigma_{\min }$ in Eq. (5) is obtained with the tightcoupling condition, $\sigma_{\min }$ actually serves as the overall lower bound for entropy production $\sigma$ with arbitrary $q$. This is because $\sigma$ decreases monotonically with the increase of $|q|$ (See SM [35] for strict proof). Therefore, for general cases within the linear response regime, the uniform temperature is bounded from below by the minimal entropy production as $\tilde{T} \geq \tilde{T}\left(\sigma_{\min }\right)$.

Trade-off between power and efficiency. - The work output in the whole process is $W(\tau)=Q_{h}(\tau)-Q_{c}(\tau)$, where $Q_{h}(\tau)=C_{h}\left(T_{h}^{[i]}-\tilde{T}\right)$ and $Q_{c}(\tau)=C_{c}\left(\tilde{T}-T_{c}^{[i]}\right)$. The maximum extractable work $W_{\max } \equiv \lim _{\sigma \rightarrow 0} W(\tau)$ [35] is achieved in the reversible case. Note that $W(\tau)$ is a monotonically decreasing function of $\tilde{T}$ [35], which indicates that, referring to Eq. (2), the entropy production will reduce $W(\tau)$ in comparison with $W_{\max }$. In this sense, we define the finite-time dissipative work

$$
W_{d} \equiv W_{\max }-W(\tau)=\left(C_{h}+C_{c}\right)\left(\tilde{T}-T_{b}\right) .
$$

It follows from Eqs. (2), (5), and (6) that the constraint on dissipative work is explicitly obtained as $W_{d} \geq$ $T_{b} \Sigma_{\min } / \tau \equiv W_{d}^{(\min )}$ [35]. In terms of $W_{d}$, the efficiency in the finite-time case, $\eta \equiv W(\tau) / Q_{h}(\tau)$, reads

$$
\eta=\frac{W_{\max }-W_{d}}{W_{\max } / \eta_{\mathrm{MW}}-W_{d} /(1+\gamma)}
$$

where the efficiency at maximum work (EMW) [35]

$$
\eta_{\mathrm{MW}} \equiv 1-\gamma\left[\frac{\eta_{\mathrm{C}}}{1-\left(1-\eta_{\mathrm{C}}\right)^{\gamma /(\gamma+1)}}-1\right]
$$

is achieved in the reversible case [33]. And $\eta_{\mathrm{C}} \equiv 1-$ $T_{c}^{[i]} / T_{h}^{[i]}$ is the Carnot efficiency determined by the initial temperatures of the reservoirs. 


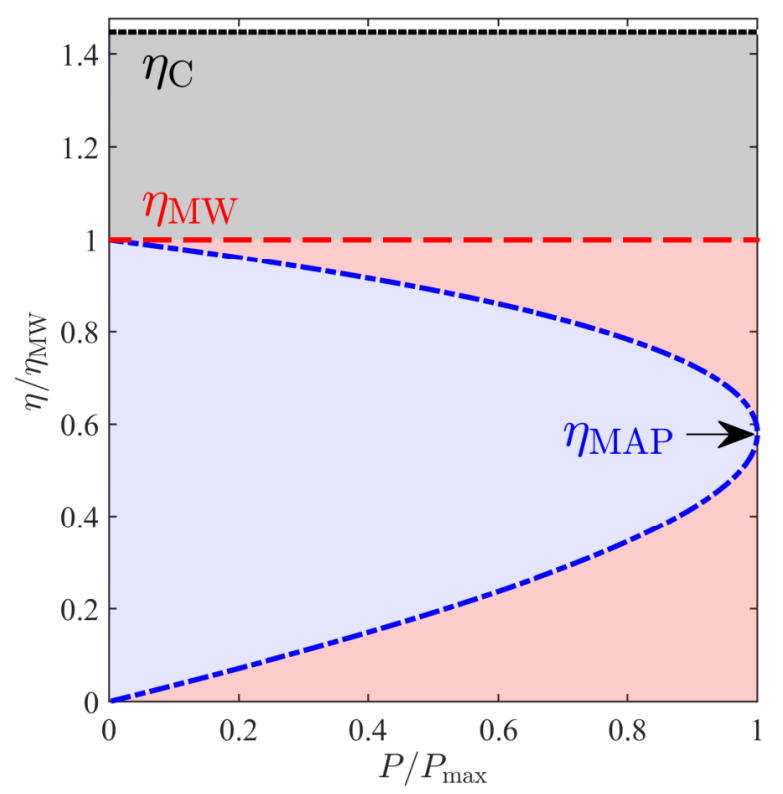

Figure 2. "Phase diagram" $\tilde{P}-\tilde{\eta}$ of the heat engine performance between finite reservoirs. The blue dash-dotted curve and the (light blue) area therein represent the trade-off between $\tilde{P}=P / P_{\max }$ and $\tilde{\eta}=\eta / \eta_{\mathrm{MW}}$ in Eq. (10). $P_{\max }$ is the maximum average power. The efficiency at maximum work $\eta_{\mathrm{MW}}$ in Eq. (8) is plotted with the red dashed line, while the corresponding Carnot efficiency $\eta_{\mathrm{C}}=0.8$ is plotted with the black dotted line. In this example, we use $\gamma=C_{c} / C_{h}=1$.

Expressing $W_{d}$ in terms of $\eta$ according to Eq. (7), the constraint on dissipative work $\left(W_{d} \geq W_{d}^{(\min )}\right)$ becomes,

$$
W_{d}=\frac{W_{\max }\left(\eta_{\mathrm{MW}}-\eta\right)}{\eta_{\mathrm{MW}}[1-\eta /(1+\gamma)]} \geq \frac{T_{b} \Sigma_{\min }}{\tau}
$$

Eliminating the duration $\tau$ in this inequality with the average power $P \equiv W(\tau) / \tau$ of the whole process, we find the trade-off relation between power and efficiency [35]

$$
\tilde{P} \leq \frac{4 \lambda \tilde{\eta}(1-\tilde{\eta})}{(\lambda \tilde{\eta}+1-\tilde{\eta})^{2}}
$$

Here, $\lambda \equiv 1-\eta_{\mathrm{MW}} /(1+\gamma), \tilde{P} \equiv P / P_{\max }, \tilde{\eta} \equiv \eta / \eta_{\mathrm{MW}}$, and $P_{\max } \equiv W_{\max }^{2} /\left(4 T_{b} \Sigma_{\min }\right)$ is the maximum average power. As the main result of this paper, the above relation specifics the complete optimization regime for the heat engines operating between finite-sized reservoirs. The equal sign of Eq. (10) is achieved with the minimum entropy generation $\sigma_{\min }$, which determines the optimal performance of the heat engine, namely, the maximum power for a given efficiency. The optimal operation of the heat engine will be discussed later. We emphasize that such a trade-off constrains the performance of all the heat engines operating in the linear response regime, because $\sigma_{\min }$ is the overall lower bound for irreversibility as we remarked below Eq. (5).

In the symmetric case with $\gamma=1$ (See SM [35] for the asymmetric cases with $\gamma=0.01,100)$, the powerefficiency trade-off is illustrated in Fig. 2 with the blue dash-dotted curve and the (light blue) area therein. The efficiency corresponding to the maximum power $(\tilde{P}=1)$ is denoted as $\eta_{\mathrm{MAP}}$ in this figure, and will be detailed discussed in the following. $\eta_{\mathrm{C}}=0.8$ is used in this plot. Due to the finiteness of the heat reservoirs, the (gray) area between efficiency at maximum work $\eta_{\mathrm{MW}}$ (red dashed line) and Carnot efficiency $\eta_{\mathrm{C}}$ (black dotted line) becomes a forbidden regime in the "phase diagram" of the heat engine performance. Particularly, in the limit of $\gamma \rightarrow \infty$ with infinite cold reservoir, the trade-off in Eq. (10) reduces to a concise form $\tilde{P} \leq 4 \tilde{\eta}(1-\tilde{\eta})$.

With the obtained power-efficiency trade-off, it is straightforward to find the efficiency at an arbitrary given power $\tilde{P}$ being bounded in the region of $\tilde{\eta}_{-} \leq \tilde{\eta} \leq \tilde{\eta}_{+}$, where $\tilde{\eta}_{ \pm}$are defined as [35]

$$
\tilde{\eta}_{ \pm} \equiv 1-\frac{\lambda \tilde{P}}{(1 \pm \sqrt{1-\tilde{P}})^{2}+\lambda \tilde{P}} .
$$

The upper bound $\tilde{\eta}_{+}$, serving as the maximum efficiency for an arbitrary average power, returns to its counterpart in the infinite-reservoir case by replacing $\eta_{\mathrm{MW}}$ with $\eta_{\mathrm{C}}$ [19, 20, 23]. Obviously, $\tilde{\eta}_{+}$approaches 1 in the quasistatic regime of $\tilde{P} \rightarrow 0$, namely, $\eta \rightarrow \eta_{\mathrm{MW}}$, as shown in Fig. 2.

Efficiency at maximum average power. - When the heat engine achieve its maximum average power $(\tilde{P}=1)$, the upper and lower bound in Eq. (11) converge to the efficiency at maximum average power (EMAP)

$$
\eta_{\mathrm{MAP}}=\frac{\eta_{\mathrm{MW}}}{2-\eta_{\mathrm{MW}} /(1+\gamma)} .
$$

We note that this general EMAP recovers $\eta_{\mathrm{MW}} / 2(\gamma \rightarrow$ $\infty)$ which was previously obtained in the special case with infinite large cold reservoir [29]. Since $\gamma \in[0, \infty]$, $\eta_{\text {MAP }}$ satisfies

$$
\eta_{\mathrm{L}} \equiv \frac{\eta_{\mathrm{MW}}}{2} \leq \eta_{\mathrm{MAP}} \leq \frac{\eta_{\mathrm{MW}}}{2-\eta_{\mathrm{MW}}} \equiv \eta_{\mathrm{U}}
$$

where the upper bound $\eta_{\mathrm{U}}$ is reached in the limit $\gamma \rightarrow 0$ $\left(C_{c} \ll C_{h}\right)$ with infinite large hot reservoir.

Figure $3\left(\right.$ a) shows the dependence of $\eta_{\mathrm{MAP}}$ on $\eta_{\mathrm{C}}$, where the (light red) area between $\eta_{\mathrm{U}}$ (red dash-dotted curve) and $\eta_{\mathrm{L}}$ (red dotted curve) is the available range of $\eta_{\mathrm{MAP}}$. In comparison, the achievable range of $\eta_{\mathrm{MW}}$ is represented with the (gray) area between the black solid curve and the black dashed curve. As demonstrated in this figure, in the small- $\eta_{\mathrm{C}}$ regime, there exist $\gamma$-independent scalings for $\eta_{\mathrm{MAP}}$ and $\eta_{\mathrm{MW}}$. Such universalities can be explicitly obtained by expanding $\eta_{\text {MAP }}$ and $\eta_{\mathrm{MW}}$ with respect to $\eta_{\mathrm{C}}$ : 
(a)

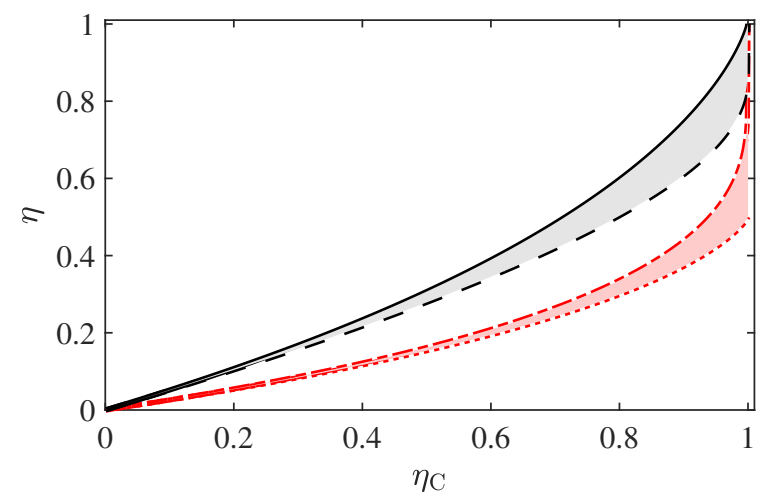

(b)

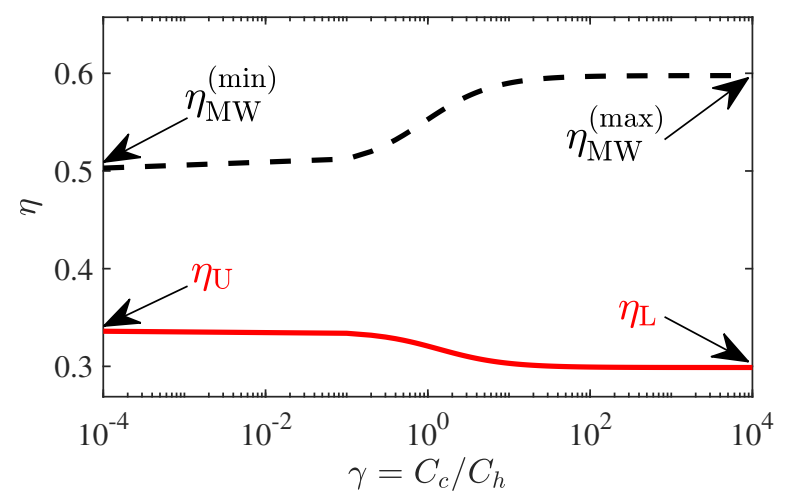

Figure 3. Dependence of and $\eta_{\mathrm{MAP}}$ and $\eta_{\mathrm{MW}}$ on $\eta_{\mathrm{C}}$ and $\gamma$. (a) $\eta_{\text {MAP }}$ and $\eta_{\mathrm{MW}}$ as the function of $\eta_{\mathrm{C}}$. The upper (lower) bound $\eta_{\mathrm{U}}\left(\eta_{\mathrm{L}}\right)$ of $\eta_{\mathrm{MAP}}$ in Eq. (13) is plotted as the red dashdotted (dotted) curve, the (light red) area between the dashdotted curve and dotted curve is the available range of $\eta_{\text {MAP }}$. The upper (lower) bound of $\eta_{\mathrm{MW}}$ is represented by the black solid (dashed) curve, and the (gray) area between the solid curve and dashed curve is the achievable range of $\eta_{\mathrm{MW}}$. (b) $\eta_{\text {MAP }}$ and $\eta_{\text {MW }}$ as the function of $\gamma$. The red solid curve and black dashed curve represent $\eta_{\mathrm{MAP}}$ and $\eta_{\mathrm{MW}}$, respectively. In this example, $\eta_{\mathrm{C}}=0.8$.

$$
\begin{aligned}
\eta_{\mathrm{MW}} & =\frac{1}{2} \eta_{\mathrm{C}}+\frac{1}{6}\left(1-\frac{1 / 2}{\gamma+1}\right) \eta_{\mathrm{C}}^{2}+\mathcal{O}\left(\eta_{\mathrm{C}}^{3}\right), \\
\eta_{\mathrm{MAP}} & =\frac{1}{4} \eta_{\mathrm{C}}+\frac{1}{12}\left(1+\frac{1 / 4}{\gamma+1}\right) \eta_{\mathrm{C}}^{2}+\mathcal{O}\left(\eta_{\mathrm{C}}^{3}\right) .
\end{aligned}
$$

Obviously, the first-order coefficients of both $\eta_{\mathrm{MW}}$ and $\eta_{\text {MAP }}$ are independent of the heat capacity ratio $\gamma$, as we inferred from Fig. 3(a). Up to the first order of $\eta_{\mathrm{C}}$, the universality of $\eta_{\mathrm{MAP}}$ scales as $\eta_{\mathrm{MAP}} \sim \eta_{\mathrm{C}} / 4$. Meanwhile, the universality of $\eta_{\mathrm{MW}}$ follows as $\eta_{\mathrm{MW}} \sim \eta_{\mathrm{C}} / 2$, which has also been revealed in previous studies [33, 34]. Nevertheless, the coefficients corresponding to the second order of $\eta_{\mathrm{C}}$ are $\gamma$-dependent for $\eta_{\mathrm{MAP}}$ and $\eta_{\mathrm{MW}}$. The signs of the terms containing $\gamma$ in $\eta_{\mathrm{MAP}}$ and $\eta_{\mathrm{MW}}$ are opposite, which indicates that the monotonicity of $\eta_{\text {MAP }}$ and $\eta_{\text {MW }}$ with respect to $\gamma$ is opposite. This fact is clearly illustrated in Fig. 3(b), where $\eta_{\mathrm{MAP}}$ (red solid curve) is a monotonically decreasing function of $\gamma$, while $\eta_{\text {MW }}$ (black dashed curve) increases with $\gamma$ monotonically [34]. In this figure, the maximum $\eta_{\mathrm{MW}}\left(\eta_{\mathrm{MW}}^{(\max )}\right)$ and minimum $\eta_{\mathrm{MW}}\left(\eta_{\mathrm{MW}}^{(\mathrm{min})}\right)$ are reached in the limit $\gamma \rightarrow \infty$ and $\gamma \rightarrow 0$, respectively [33], and $\eta_{\mathrm{C}}=0.8$ is fixed. As the result of the opposite monotonicity, there exists a competitive relation between $\eta_{\mathrm{MAP}}$ and $\eta_{\mathrm{MW}}$. Namely, $\eta_{\mathrm{MAP}}$ achieves its maximum even when $\eta_{\mathrm{MW}}$ is minimum in the limit $\gamma \rightarrow 0$, and vice versa.

Optimal operation protocol of the heat engine. - As a process function, the path dependence of entropy production $\sigma$ in the parameter space makes it relies on the control protocol applied to the working substance [43, 44]. Therefore, the efficiency and power of the heat engine are inseparable from the specific operation protocol of the cycle. To achieve the boundary of the trade-off (10) or the EMAP (12), we demonstrate the optimal operation of the heat engine associated with the minimal entropy production $\sigma_{\min }$ with a specific example. For a finite-time Carnot heat engine whose working substance is the ideal gas with volume $V$ (control parameter), the minimal entropy production condition, i.e., $\dot{Q}_{h}=\sqrt{L_{22} \Sigma_{\min }} / \tau$, allows us to find the optimal control protocol for $V(t)$ from the energy conservation relation of the gas [35].

The optimal operation protocol of the heat engine is shown in Fig. 4, where A (C) represents the finitetime isothermal expansion (compression) process with duration $t_{h}^{(m)}\left(t_{c}^{(m)}\right)$ in the $m$-th $(m=1,2,3 \ldots M)$ cycle. During the isothermal expansion (compression), the gas volume changes exponentially with time as $V_{h}^{(m)}(\tilde{t})=$ $V_{h, i}^{(m)} \exp \left(\Gamma_{h}^{(m)} \tilde{t}\right) \quad\left(V_{c}^{(m)}\left(t^{\prime}\right)=V_{c, i}^{(m)} \exp \left(-\Gamma_{c}^{(m)} t^{\prime}\right)\right)$ with $\tilde{t} \equiv t-(m-1) \tau_{c}\left(t^{\prime} \equiv t-(m-1) \tau_{c}-t_{h}^{(m)}\right)$. Here, the initial volume of the gas in the isothermal expansion process $V_{h, i}^{(m)}=V_{h, i}$ is fixed in each cycle, while the initial volume of other three processes $\left(V_{h, f}^{(m)}, V_{c, i}^{(m)}\right.$, and $\left.V_{c, f}^{(m)}\right)$ are determined by the full operation protocol. $\Gamma_{h(c)}^{(m)}$ represents the isothermal expansion (compression) rate of the $m$-th cycle [35]. The adiabatic equation of ideal gas is satisfied in the adiabatic processes $\mathrm{B}$ and $\mathrm{D}$, the duration of which is ignored in comparison with that of the isothermal processes $[15,44,45]$. It is worth mentioning that a recent study [46] obtained similar optimal operation to realize the efficiency at maximum power of a Brownian heat engine between constant temperature reservoirs. This reminds us that such an optimal operation scheme may be universal for some types of finite-time heat engines.

Conclusion and discussion-In summary, we successfully obtained a general power-efficiency trade-off for heat engines operating between two finite-sized reservoirs within the linear response regime. With such a trade-off, we showed the achievable range of efficiency for a given average power, and the universal efficiency at maximum 


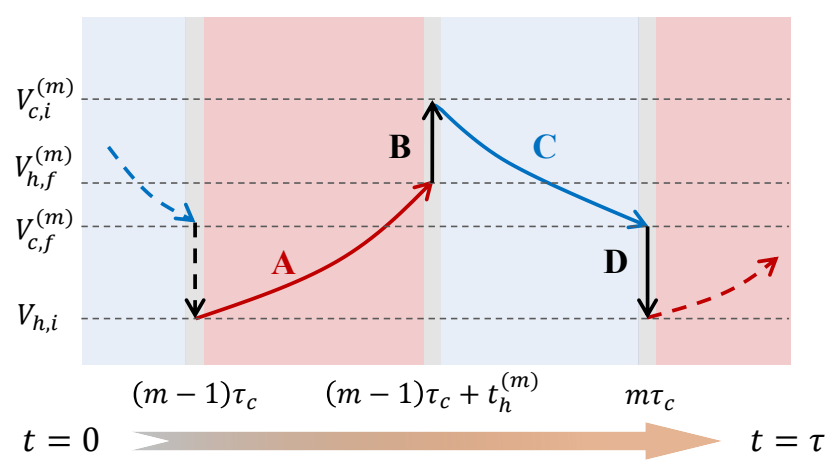

Figure 4. The diagram of the optimal operation protocol of the $m$-th cycle with the control parameter $V$ (gas volume). In the isothermal expansion (expression) process $A(C)$ of duration $t_{h}\left(t_{c}\right), V$ changes exponentially with time; while in adiabatic processes (B and $\mathrm{D}), V$ is quenched with the adiabatic equation of idea gas being satisfied.

average power. To achieve the optimal performance of the heat engine, corresponding to the boundary of the power-efficiency trade-off, the optimal operation protocol of an ideal gas heat engine is demonstrated. The predicted results can be tested on some state-of-art platforms $[44,47]$. Moreover, by replacing $\eta_{\mathrm{MW}}$ with $\eta_{\mathrm{C}}$, some typical constraints in finite case become their corresponding counterparts in infinite case. These thermodynamic constraints specific the full operation regime of the heat engines in real-world circumstances. Basically, this study paves the way for the joint optimization of thermodynamic cycle by adjusting the ratio of the heat capacities of the reservoirs and controlling the operation of the cycle, and may shed new light on investigating the irreversibility of non-equilibrium thermodynamic processes off thermodynamic limit.

The temperature-dependent feature of the reservoir's heat capacity [33], the quantumness of the reservoir [48$50]$, the deviation of entropy production from $1 / \tau$-scaling beyond the slow-driving regime [23, 43, 44, 51], and the fluctuations in heat engine performance [52-54] are expected to be taken into future considerations.

Acknowledgment.-We thank G. H. Dong and Y. Chen for the helpful suggestions. We are grateful to the anonymous referees for enlightening comments. This work is supported by the National Science Foundation of China (NSFC) (Grants No. 11534002, No. 11875049, No. U1730449, No. U1530401, and No. U1930403), the National Basic Research Program of China (Grants No. 2016YFA0301201), and the China Postdoctoral Science Foundation (Grant No. BX2021030).
[1] K. Huang, Introduction To Statistical Physics, 2Nd Edition (T\&F/Crc Press, 2013), ISBN 978-1-4200-7902-9.

[2] B. Andresen, R. S. Berry, M. J. Ondrechen, and P. Salamon, Acco. Chem. Res. 17, 266 (1984).

[3] U. Seifert, Rep. Prog. Phys. 75, 126001 (2012).

[4] V. Holubec and A. Ryabov, Phys. Rev. E 96 (2017).

[5] R. Kosloff, J. Chem. Phys. 150, 204105 (2019).

[6] Z.-C. Tu, Front. Phys. 16, 1 (2021).

[7] J. Yvon, in First Geneva Conf. Proc. UN (1955).

[8] P. Chambadal, Recuperation de chaleura la sortie d' un reacteur, chapter 3 (1957).

[9] I. I. Novikov, J. Nucl. Energy II 7, 125 (1958).

[10] F. L. Curzon and B. Ahlborn, Am. J. Phys. 43, 22 (1975).

[11] C. V. den Broeck, Phys. Rev. Lett. 95, 190602 (2005).

[12] Y. Izumida and K. Okuda, EPL 83, 60003 (2008).

[13] T. Schmiedl and U. Seifert, EPL 83, 30005 (2008).

[14] Z. C. Tu, Journal Phys. A: Math. Theor. 41, 312003 (2008).

[15] M. Esposito, R. Kawai, K. Lindenberg, and C. V. den Broeck, Phys. Rev. Lett. 105, 150603 (2010).

[16] Y. Wang and Z. C. Tu, Phys. Rev. E 85, 011127 (2012).

[17] C. V. D. Broeck, EPL 101, 10006 (2013).

[18] V. Holubec and A. Ryabov, Phys. Rev. E 92, 052125 (2015).

[19] V. Holubec and A. Ryabov, J. Stat. Mech. Theo. Exp. 2016, 073204 (2016).

[20] R. Long and W. Liu, Phys. Rev. E 94, 052114 (2016).

[21] N. Shiraishi, K. Saito, and H. Tasaki, Phys. Rev. Lett. 117, 190601 (2016).

[22] V. Cavina, A. Mari, and V. Giovannetti, Phys. Rev. Lett.
119, 050601 (2017).

[23] Y.-H. Ma, D. Xu, H. Dong, and C.-P. Sun, Phys. Rev. E 98, 042112 (2018).

[24] A. C. Barato and U. Seifert, Phys. Rev. Lett. 114, 158101 (2015).

[25] J. M. Horowitz and T. R. Gingrich, Phys. Rev. E 96, 020103(R) (2017).

[26] M. J. Ondrechen, B. Andresen, M. Mozurkewich, and R. S. Berry, Am. J. Phys. 49, 681 (1981).

[27] M. J. Ondrechen, M. H. Rubin, and Y. B. Band, J. Chem. Phys. 78, 4721 (1983).

[28] H. S. Leff, Am. J. Phys. 55, 701 (1987).

[29] Y. Izumida and K. Okuda, Phys. Rev. Lett. 112, 180603 (2014).

[30] Y. Wang, Phys. Rev. E 90, 062140 (2014).

[31] R. S. Johal, Phys. Rev. E 94, 012123 (2016).

[32] H. Tajima and M. Hayashi, Phys. Rev. E 96, 012128 (2017).

[33] Y.-H. Ma, Entropy 22, 1002 (2020).

[34] R. S. Johal and R. Rai, EPL 113, 10006 (2016).

[35] See Supplemental Materials for detailed discussion on the reversible regime (Sec. I); the lower bound of irreversible entropy production (Sec. II); the dissipation work and power-efficiency trade-off (Sec. III); the bounds of $\tilde{\eta}_{ \pm}$ (Sec. IV); and the optimal operation of the engine (Sec. $\mathrm{V})$.

[36] Y. Izumida and K. Okuda, Phys. Rev. E 80, 021121 (2009).

[37] K. Proesmans and C. Van den Broeck, Phys. Rev. Lett. 115, 090601 (2015). 
[38] Y. Izumida, Phys. Rev. E 103, L050101 (2021).

[39] G. Ruppeiner, Phys. Rev. A 20, 1608 (1979).

[40] P. Salamon and R. S. Berry, Phys. Rev. Lett. 51, 1127 (1983).

[41] G. E. Crooks, Phys. Rev. Lett. 99 (2007).

[42] K. Sekimoto and S. ichi Sasa, Journal of the Phys. Society of Japan 66, 3326 (1997).

[43] Y.-H. Ma, D. Xu, H. Dong, and C.-P. Sun, Phys. Rev. E 98, 022133 (2018).

[44] Y.-H. Ma, R.-X. Zhai, J. Chen, H. Dong, and C. P. Sun, Phys. Rev. Lett. 125, 210601 (2020).

[45] Z.-C. Tu, Chin. Phys. B 21, 020513 (2012).

[46] Y.-H. Chen, J.-F. Chen, Z. Fei, and H.-T. Quan, arXiv:2108.04128 (2020).

[47] I. A. Martínez, É. Roldán, L. Dinis, D. Petrov, J. M. R.
Parrondo, and R. A. Rica, Nat. Phys. 12, 67 (2015).

[48] D. Xu, S.-W. Li, X. Liu, and C. Sun, Phys. Rev. E 90, 062125 (2014).

[49] J. Roßnagel, O. Abah, F. Schmidt-Kaler, K. Singer, and E. Lutz, Phys. Rev. Lett. 112 (2014).

[50] Y.-H. Ma, C. L. Liu, and C. P. Sun, arXiv:2110.04550 (2021).

[51] Y.-H. Ma, H. Dong, and C. P. Sun, Commun. Theor. Phys. 73, 125101 (2021).

[52] G. Verley, T. Willaert, C. Broeck, and M. Esposito, Phys. Rev. E 90, 052145 (2014).

[53] T. Denzler and E. Lutz, Phys. Rev. Res. 2 (2020).

[54] Z. Fei, J.-F. Chen, and Y.-H. Ma, arXiv:2109.12816 (2021). 


\title{
Supplementary Materials for "Optimizing Thermodynamic cycles with Finite-Sized Reservoirs"
}

\author{
Hong Yuan, ${ }^{1}$ Yu-Han Ma, ${ }^{1, *}$ and C. P. Sun ${ }^{1,2, \dagger}$ \\ ${ }^{1}$ Graduate School of China Academy of Engineering Physics, \\ No. 10 Xibeiwang East Road, Haidian District, Beijing, 100193, China \\ ${ }^{2}$ Beijing Computational Science Research Center, Beijing 100193, China
}

This document is devoted to providing the detailed derivations and the supporting discussions to the main content of the Letter. In Sec. I, we discuss the final uniform temperature $\tilde{T}$, the maximum work $W_{\max }$, and the corresponding efficiency $\eta_{\mathrm{MW}}$ in the reversible regime under the quasi-static limit. The lower bound of irreversible entropy production, illustrated in [Eq. (5)] of the main text, is derived in Sec. II. In Sec. III, we show the proofs of the lower bound of dissipative work $W_{d} \geq T_{b} \Sigma_{\min } / \tau$, and the trade-off between power and efficiency presented in [Eq. (10)] of the main text. The derivation of the bounds for efficiency at arbitrary given power $\tilde{\eta}_{ \pm}$[Eq. (11) of the main text] is given in Sec. IV. In Sec. V, taking idea gas heat engine as an example, we demonstrate the optimal operation protocol of the engine associated with the minimal entropy production to achieve the boundary of the power-efficiency trade-off.

\section{THE REVERSIBLE REGIME}

The extractable work from the reservoirs in the reversible regime, $W_{\max } \equiv \lim _{\sigma \rightarrow 0} W(\tau)$, is the upper bound of the work output until the heat engines stop operating. Such bound is achieved with no irreversible entropy production in the whole process [1-3]. Specifically, in an infinitesimal process, The heat engine absorbs $d Q_{h}=-C_{h} d T_{h}$ from the hot reservoir, and then transforms it into the infinitesimal work the $d W_{\max }$ with the corresponding time-dependent Carnot efficiency $\eta_{\mathrm{C}}(t) \equiv 1-T_{c} / T_{h}[2]$. In this sense, the power of the engine reads

$$
d W_{\max }=-C_{h} d T_{h}\left(1-\frac{T_{c}}{T_{h}}\right) .
$$

According to the conservation of energy $d W_{\max }=d Q_{h}-d Q_{c}$, we can also write

$$
d W_{\max }=-C_{h} d T_{h}-C_{c} d T_{c},
$$

where $d Q_{c}=C_{c} d T_{c}$ is the heat released from the heat engine to the cold reservoir. Comparing Eq. (1) and Eq. (2), one has

$$
-C_{h} \frac{d T_{h}}{T_{h}}=C_{c} \frac{d T_{c}}{T_{c}}
$$

In the case with constant heat capacity $C_{h}\left(C_{c}\right)$, by integrating both sides of Eq. (3), the final uniform temperature of the total system, $T_{b} \equiv T_{c}^{[f]}=T_{h}^{[f]}$, is obtained as

$$
T_{b}=\left[T_{h}^{[i]}\right]^{\frac{1}{\gamma+1}}\left[T_{c}^{[i]}\right]^{\frac{\gamma}{\gamma+1}}
$$

where $\gamma \equiv C_{c} / C_{h}$ is defined as the heat capacity ratio. As a consequence, integrating Eq. (2), we find the maximum work output

$$
W_{\max }=C_{h}\left(T_{h}^{[i]}-T_{b}\right)-C_{c}\left(T_{b}-T_{c}^{[i]}\right) .
$$

The first term of the right hand of Eq. (5) is the reversible heat absorbed, $Q_{h}(\sigma \rightarrow 0)=C_{h}\left[T_{h}^{[i]}-T_{b}\right]$, achieved in the quasi-static limit. In this case, the corresponding efficiency, namely, the efficiency at maximum work output $(\mathrm{EMW}) \eta_{\mathrm{MW}} \equiv W_{\max } / Q_{h}(\sigma \rightarrow 0)$, is directly obtained as [3]

$$
\eta_{\mathrm{MW}}=1-\gamma\left[\frac{\eta_{\mathrm{C}}}{1-\left(1-\eta_{\mathrm{C}}\right)^{\frac{\gamma}{\gamma+1}}}-1\right],
$$

\footnotetext{
*yhma@gscaep.ac.cn

† suncp@gscaep.ac.cn
} 
where $\eta_{\mathrm{C}} \equiv \eta_{\mathrm{C}}(0)=1-T_{c}^{[i]} / T_{h}^{[i]}$ is the Carnot efficiency determined by the initial temperature of the two reservoirs. As we mentioned before, this result is applicable to the heat reservoirs with constant heat capacity. For the reservoirs with temperature-dependent heat capacity, the generalization of Eq. (6) is studied our previous work [3].

\section{LOWER BOUND OF IRREVERSIBLE ENTROPY PRODUCTION}

It follows from [Eq. (3)] and [Eq. (4)] of the main text that the entropy production $\sigma(\tau)=\int_{0}^{\tau}(\sqrt{\dot{\sigma}})^{2} d t$ explicitly follows as

$$
\begin{aligned}
\sigma(\tau) & \geq \frac{\left(\int_{0}^{\tau} C_{h} \dot{T}_{h} / \sqrt{L_{22}} d t\right)^{2}}{\tau}, \\
& =\frac{\left(\int_{T_{h}^{[i]}}^{\tilde{T}} C_{h} d T_{h} / \sqrt{L_{22}}\right)^{2}}{\tau} \\
& \equiv \frac{\Sigma}{\tau}
\end{aligned}
$$

where $\Sigma=\Sigma(\tilde{T})$, and $T_{h}(\tau) \equiv \tilde{T}=\tilde{T}(\sigma)$ is given by [Eq. (2)] of the main text. In the linear irreversible regime, keeping the first order of $\sigma$ in $\tilde{T}(\sigma),[\mathrm{Eq} .(2)]$ of the main text is approximated as

$$
\begin{aligned}
\tilde{T} & =T_{b} \exp \left[\frac{\sigma}{C_{h}+C_{c}}\right] \\
& \approx T_{b}\left[1+\frac{\sigma}{C_{h}+C_{c}}\right] .
\end{aligned}
$$

Then, $\Sigma$ is approximated as

$$
\begin{aligned}
\Sigma & =\Sigma\left(\tilde{T}=T_{b}\right)+\left.\frac{\partial \Sigma}{\partial \tilde{T}}\right|_{\tilde{T}=T_{b}}\left(\tilde{T}-T_{b}\right) \\
& =\left(\int_{T_{h}^{[i]}}^{T_{b}} C_{h} d T_{h} / \sqrt{L_{22}}\right)^{2}+2\left(\int_{T_{h}^{[i]}}^{T_{b}} C_{h} d T_{h} / \sqrt{L_{22}}\right) \frac{T_{b} \sigma}{\sqrt{L_{22}}(1+\gamma)},
\end{aligned}
$$

up to the first order of $\sigma$. Substituting the above result into Eq. (9), we obtain

$$
\sigma(\tau) \geq \frac{\Sigma_{\min }}{\tau}\left[1-\frac{2 T_{b} \sqrt{\Sigma_{\min } / L_{22}}}{(1+\gamma) \tau}\right]^{-1}
$$

where $\Sigma_{\min } \equiv\left(\int_{T_{h}^{[i]}}^{T_{b}} C_{h} d T_{h} / \sqrt{L_{22}}\right)^{2}$ is a $\tau$-independent dissipation coefficient. By expanding the right hand of this inequality with respect to $\tau^{-1}$, we have

$$
\sigma(\tau) \geq \frac{\Sigma_{\min }}{\tau}\left[1+\frac{2 T_{b} \sqrt{\Sigma_{\min } / L_{22}}}{(1+\gamma) \tau}\right]+\mathcal{O}\left(\tau^{-3}\right)
$$

In the long-time regime, up to the first order of $\tau^{-1}$, we obtain [Eq. (5)] of the main text, namely, $\sigma(\tau) \geq \Sigma_{\text {min }} / \tau$. To be consistent with this result, all the high-order terms of $\tau^{-1}$ will be ignored in the following discussion.

It is worth mentioning that, even without the tight-coupling condition, [Eq.(5)] of the main text serves as the overall lower bound for entropy production. The proof is as follows: based on the linear irreversible thermodynamics, the entropy production rate $\dot{\sigma}$ reads [4] 


$$
\dot{\sigma}=\frac{1}{L_{22} q^{2}} J_{2}^{2}+\frac{1-q^{2}}{q^{2}} L_{22} X_{2}^{2}-2 \frac{1-q^{2}}{q^{2}} J_{2} X_{2}
$$

Here $J_{2} \equiv \dot{Q}_{h}$ is the heat flow from the hot reservoir, $X_{2} \equiv 1 / T_{c}-1 / T_{h}$ represents the conjugate thermodynamic force, and the coefficient of the coupling strength $q \equiv L_{21} / \sqrt{L_{11} L_{22}}$ satisfies $|q| \leq 1$. The derivative of $\sigma$ with respect to $q^{2}$ is straightforward obtained as

$$
\begin{aligned}
\frac{\partial \sigma}{\partial\left(q^{2}\right)} & =\int_{0}^{\tau} \frac{\partial \dot{\sigma}}{\partial\left(q^{2}\right)} d t \\
& =-\int_{0}^{\tau} \frac{1}{q^{4}}\left(\frac{J_{2}}{\sqrt{L_{22}}}-\sqrt{L_{22}} X_{2}\right)^{2} d t \leq 0
\end{aligned}
$$

which is always negative. This indicates that the entropy production decreases monotonically with the increase of $|q|$. Therefore, the minimum entropy production obtained in [Eq.(5)] of the main text serves as the overall lower bound for entropy production with arbitrary $q$.

\section{DISSIPATIVE WORK AND POWER-EFFICIENCY TRADE-OFF}

\section{A. The dissipative work}

The work output $W(\tau)=Q_{h}(\tau)-Q_{c}(\tau)$ is explicitly written as

$$
W(\tau)=C_{h}\left(T_{h}^{[i]}-\tilde{T}\right)-C_{c}\left(\tilde{T}-T_{c}^{[i]}\right)
$$

which is monotonically decreasing as $\tilde{T}$ increases. It follows from Eqs. (5) and (19) that the dissipative work $W_{d}(\tau) \equiv W_{\max }-W(\tau)$ reads

$$
W_{d}(\tau)=\left(C_{c}+C_{h}\right)\left(\tilde{T}-T_{b}\right)
$$

Combining [Eq. (2)] and [Eq. (5)] of the main text, one has

$$
\tilde{T} \geq T_{b} \exp \left[\frac{\Sigma_{\min }}{\left(C_{c}+C_{h}\right) \tau}\right]
$$

and then Eq. (20) is written in terms of $\tau$ as,

$$
\begin{aligned}
W_{d}(\tau) & \geq\left(C_{h}+C_{c}\right) T_{b}\left[e^{\frac{\Sigma_{\min }}{\left(C_{c}+C_{h}\right) \tau}}-1\right] \\
& =\frac{T_{b} \Sigma_{\min }}{\tau}+\mathcal{O}\left(\tau^{-2}\right)
\end{aligned}
$$

Up to the first order of $\tau^{-1}$, the inequality for the dissipative work in main text is obtained as $W_{d}(\tau) \geq T_{b} \Sigma_{\min } / \tau$.

\section{B. Power-efficiency trade-off}

As shown in [Eq. (9)] of the main text that

$$
\frac{\eta_{\mathrm{MW}}-\eta}{\eta_{\mathrm{MW}}[1-\eta /(1+\gamma)]} \geq \frac{T_{b} \Sigma_{\min }}{W_{\max } \tau}
$$


which becomes, by multiplying both sides by $W(\tau)$,

$$
\begin{aligned}
\frac{W(\tau)\left(\eta_{\mathrm{MW}}-\eta\right)}{\eta_{\mathrm{MW}}[1-\eta /(1+\gamma)]} & \geq \frac{T_{b} \Sigma_{\min } W(\tau)}{W_{\max } \tau} \\
& =\frac{T_{b} \Sigma_{\min } P}{W_{\max }}
\end{aligned}
$$

Here, $P \equiv W(\tau) / \tau$ is defined as the average power of the whole process. Further, we rewrite $W(\tau)$ as

$$
\begin{aligned}
W(\tau) & =W_{\max }-W_{d} \\
& =W_{\max }-\frac{W_{\max }\left(\eta_{\mathrm{MW}}-\eta\right)}{\eta_{\mathrm{MW}}[1-\eta /(1+\gamma)]}
\end{aligned}
$$

then Eq. (26) becomes

$$
W_{\max }\left\{1-\frac{\eta_{\mathrm{MW}}-\eta}{\eta_{\mathrm{MW}}[1-\eta /(1+\gamma)]}\right\} \frac{\left(\eta_{\mathrm{MW}}-\eta\right)}{\eta_{\mathrm{MW}}[1-\eta /(1+\gamma)]} \geq \frac{T_{b} \Sigma_{\min } P}{W_{\max }} .
$$

By straightforward calculation, the above inequality is simplified as

$$
P \leq \frac{W_{\max }^{2}}{\eta_{\mathrm{MW}}^{2} T_{b} \Sigma_{\min }} \frac{\eta\left[1-\eta_{\mathrm{MW}} /(1+\gamma)\right]\left(\eta_{\mathrm{MW}}-\eta\right)}{[1-\eta /(1+\gamma)]^{2}}
$$

On the other hand, the average power $P=W(\tau) / \tau$ follows as

$$
\begin{aligned}
P & =\frac{W_{\max }-W_{d}}{\tau} \\
& \leq \frac{W_{\max }-T_{b} \Sigma_{\min } / \tau}{\tau}
\end{aligned}
$$

where $W_{d} \geq T_{b} \Sigma_{\min } / \tau$ has been used. The above relation can be further written in the quadratic form, namely,

$$
P \leq-T_{b} \Sigma_{\min }\left(\frac{1}{\tau}-\frac{W_{\max }}{2 T_{b} \Sigma_{\min }}\right)^{2}+\frac{W_{\max }^{2}}{4 T_{b} \Sigma_{\min }}
$$

which clearly shows that the maximum average power and the corresponding optimal operation time are

$$
P=\frac{W_{\max }^{2}}{4 T_{b} \Sigma_{\min }} \equiv P_{\max }, \text { and } \tau=\frac{2 T_{b} \Sigma_{\min }}{W_{\max }} \equiv \tau^{*}
$$

respectively. Finally, with the definitions

$$
\tilde{P} \equiv \frac{P}{P_{\max }}, \tilde{\eta} \equiv \frac{\eta}{\eta_{\mathrm{MW}}}, \lambda \equiv 1-\frac{\eta_{\mathrm{MW}}}{1+\gamma}
$$

Eq. (30) is re-expressed as a concise form

$$
\tilde{P} \leq \frac{4 \lambda \tilde{\eta}(1-\tilde{\eta})}{(\lambda \tilde{\eta}+1-\tilde{\eta})^{2}}
$$

This is the trade-off between power and efficiency as we presented in [Eq. (10)] of the main text. In [Fig. 2] of the main text, we plot such trade-off in the symmetric case with $\gamma=C_{c} / C_{h}=1$. As comparisons, the trade-off in the asymmetric cases with $\gamma=0.01,100$ are shown in Fig. 1. 


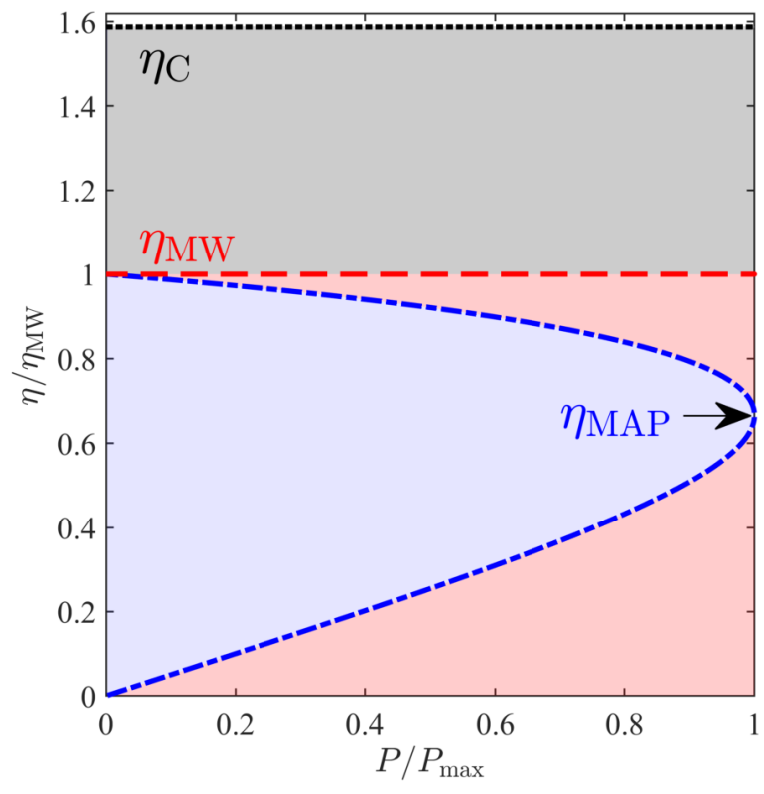

(a)

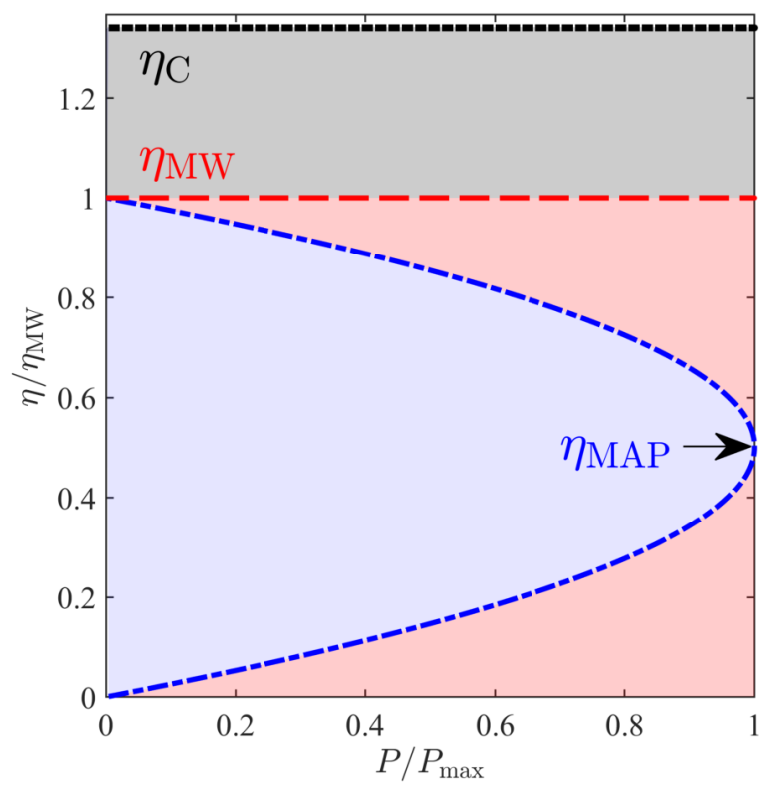

(b)

Figure 1: "Phase diagram" $\tilde{P}-\tilde{\eta}$ of the heat engine performance between finite reservoirs with different heat capacity ratio $\gamma=0.01[(\mathrm{a})]$ and $\gamma=100[(\mathrm{~b})]$. The blue dash-dotted curve and the (light blue) area therein represent the trade-off between $\tilde{P}=P / P_{\max }$ and $\tilde{\eta}=\eta / \eta_{\mathrm{MW}}$ in Eq. (36), where $P_{\max }$ is the maximum average power. The efficiency at maximum work $\eta_{\mathrm{MW}}$ in Eq. (6) is plotted with the red dashed line, while the corresponding Carnot efficiency $\eta_{\mathrm{C}}=0.8$ is plotted with the black dotted line.

\section{BOUNDS OF EFFICIENCY AT ARBITRARY GIVEN POWER}

Note that the numerator in the right side of the trade-off in Eq. (36) can be re-expressed as

$$
4 \lambda \tilde{\eta}(1-\tilde{\eta})=(\lambda \tilde{\eta}+1-\tilde{\eta})^{2}-[\lambda \tilde{\eta}-(1-\tilde{\eta})]^{2} .
$$

Thus, the trade-off is rewritten as

$$
\tilde{P}+\left[\frac{\lambda \tilde{\eta}-(1-\tilde{\eta})}{\lambda \tilde{\eta}+1-\tilde{\eta}}\right]^{2} \leq 1,
$$

such that the upper and lower bounds in $\tilde{\eta}_{-} \leq \tilde{\eta} \leq \tilde{\eta}_{+}$are the solutions of the quadratic equation

$$
\left[\frac{\lambda \tilde{\eta}-(1-\tilde{\eta})}{\lambda \tilde{\eta}+1-\tilde{\eta}}\right]^{2}=1-\tilde{P}
$$

By straightforward calculation, one has

$$
\tilde{\eta}_{ \pm}=\frac{1 \pm \sqrt{1-\tilde{P}}}{1 \pm \sqrt{1-\tilde{P}}+\lambda(1 \mp \sqrt{1-\tilde{P}})}=1-\frac{\lambda(1 \mp \sqrt{1-\tilde{P}})}{1 \pm \sqrt{1-\tilde{P}}+\lambda(1 \mp \sqrt{1-\tilde{P}})} .
$$

With the help of the relation

$$
\tilde{P}=1-(\sqrt{1-\tilde{P}})^{2}=(1 \mp \sqrt{1-\tilde{P}})(1 \pm \sqrt{1-\tilde{P}})
$$


Eq. (40) is simplified as

$$
\tilde{\eta}_{ \pm}=1-\frac{\lambda \tilde{P}}{(1 \pm \sqrt{1-\tilde{P}})^{2}+\lambda \tilde{P}} .
$$

This is the result we presented in [Eq. (11)] of the main text.

\section{OPTIMAL OPERATION PROTOCOL OF THE HEAT ENGINE}

Recent studies show that the operation protocols applied to the working substance will influence the performance of the heat engines $[5,6]$. In our model, different operation protocols of the heat engine cycle will lead to different entropy production $\sigma$, and thus affect the uniform temperature $\tilde{T}$ of the reservoirs as well as the power and efficiency of the heat engine. To achieve the boundary of the trade-off between power and efficiency, in this section, we aim to find the optimal operation protocol of the heat engine associated with the minimum entropy production $\sigma_{\min }$.

The minimum entropy production demonstrated in [Eq. (5)] of the main text is achieved with the following condition

$$
\sqrt{\dot{\sigma}}=-\frac{C_{h} \dot{T}_{h}}{\sqrt{L_{22}}}=\frac{\sqrt{\Sigma_{\min }}}{\tau} .
$$

In the case of constant $L_{22}$, one has $\Sigma_{\min }=C_{h}^{2}\left[T_{h}^{[i]}-T_{b}\right]^{2} / L_{22}=$ const, which can be simplified as $C_{h} \dot{T}_{h}=$ const. Notice that $\dot{T}_{h}$ represents the change of temperature of the hot reservoir per cycle, one has

$$
J_{2} \equiv \dot{Q}_{h}=-C_{h} \dot{T}_{h}=\frac{\sqrt{L_{22} \Sigma_{\min }}}{\tau}=\frac{C_{h}\left[T_{h}^{[i]}-T_{b}\right]}{\tau},
$$

which means that the heat absorption in each cycle is equal. On the other hand, in the tight-coupling case with $|q|=1$, the heat release to the cold reservoir follows as [2]

$$
\dot{Q}_{c}=-\frac{T_{c}}{T_{h}} C_{h} \dot{T}_{h}+\frac{T_{c}}{L_{22}}\left(C_{h} \dot{T}_{h}\right)^{2}=\frac{T_{c}}{T_{h}} \frac{C_{h}\left[T_{h}^{[i]}-T_{b}\right]}{\tau}+T_{c} \frac{C_{h}^{2}\left[T_{h}^{[i]}-T_{b}\right]^{2}}{L_{22} \tau^{2}} .
$$

Arrive here, we have obtained the heat absorption and the heat release of each cycle of the entire process.

Before proceeding further, it is worth mentioning that the time scale $\tau_{e}$, characterizing the temperature change of the reservoirs, is much greater than the mean cycle time $\tau_{c}$ (microscopic level), and therefore $T_{h(c)}$ can be regarded as constant in one cycle. Meanwhile, $\tau_{e}$ is less than the total time $\tau$ (macroscopic level), which implies that in the whole process under consideration, $T_{h(c)}$ will evolve over time. More precisely, $T_{h(c)}$ is a step function of $t$ with the intervals being the time of cycles. By taking the mean cycle time $\tau_{c}$ as the unit of time, $T_{h(c)}$ is a slowly varying function of $m \equiv t / \tau_{c},\left(m=1,2,3 \ldots M=\tau / \tau_{c}\right)$, i.e., $T_{h(c)}(t)=T_{h(c)}^{(m)}$, where the new variable $m$ represents the macroscopic dimensionless time. We stress here that $T_{h(c)}^{(m)}$ represents the temperature of the reservoir at the beginning of the corresponding finite-time isothermal process the $m$-th cycle, such that $T_{h(c)}^{(1)}=T_{h(c)}^{[i]}$. By integrating Eq.(44), the temperature of the hot reservoir in the $m$-th cycle is obtained as

$$
T_{h}^{(m)}=T_{h}^{[i]}-\frac{\left[T_{h}^{[i]}-T_{b}\right]}{M}(m-1) .
$$

Then, combining Eq.(45) and Eq.(46), one finds the temperature of the cold reservoir

$$
T_{c}^{(m)}=T_{c}^{[i]}\left[1-\frac{\left[T_{h}^{[i]}-T_{b}\right]}{M T_{h}^{[i]}}(m-1)\right]^{-\frac{1}{\gamma}} \exp \left[\frac{C_{h}^{2}\left[T_{h}^{[i]}-T_{b}\right]^{2}}{C_{c} L_{22} M \tau}(m-1)\right] .
$$

As an example, we specific the working substance of the heat engine as the ideal gas and assume that the duration of the adiabatic processes can be ignored [7]. In this case, the cycle time is approximately the sum of the time of two finite-time isothermal processes. The whole operation of the engine is illustrated in [Fig. 4] of the main text, where $m$-th cycle contains four thermodynamic processes A, B, C, and D. 
(A). In the finite-time isothermal expansion process of the $m$-th cycle with the duration $t_{h}^{(m)}$, we assume that the heat flux from high-temperature reservoir to the working substance satisfies the Newton's law [6] as

$$
q_{h}^{(m)}=\kappa_{h}\left[T_{h}^{(m)}-T_{s h}^{(m)}\right]
$$

Here, $q_{h}^{(m)}$ is the heat flow, $\kappa_{h}$ is the thermal conductivity, and $T_{s h}^{(m)}$ is the temperature of the gas which has been assumed to be unchanged in this sub-process [8]. Since the heat absorbed by the working substance from the hot reservoir in the isothermal expansion process is exactly the total heat released by the heat reservoir in $m$-th cycle, namely, $q_{h}^{(m)} t_{h}^{(m)}=\dot{Q}_{h} \tau_{c}$, the temperature of the gas is obtained as

$$
T_{s h}^{(m)}=T_{h}^{(m)}-\frac{C_{h}\left(T_{h}^{[i]}-T_{b}\right)}{M \kappa_{h} t_{h}^{(m)}} .
$$

Alternatively, with the help of the general gas equation $P V=N T_{s}$ ( $P$ is pressure of the gas, $V$ is volume of the gas, $N$ is the number of particles and we have taken $k_{B}=1$ ), the energy conservation for the gas is expressed as

$$
d U^{(m)}=q_{h}^{(m)} d t-\frac{N T_{s h}^{(m)}}{V_{h}^{(m)}} d V_{h}^{(m)} .
$$

Note that $d U^{(m)}=0$ for the idea gas with constant temperature, Eq.(50) is further simplified as

$$
\frac{N T_{s h}^{(m)}}{V} d V=q_{h}^{(m)} d t=\frac{C_{h}\left(T_{h}^{[i]}-T_{b}\right)}{M t_{h}^{(m)}} d t
$$

Integrating both sides of Eq.(51) and introducing a new time variable $\tilde{t} \equiv t-(m-1) \tau_{c} \in\left[0, t_{h}^{(m)}\right]$, we obtain the optimal protocol of the isothermal expansion as

$$
V_{h}^{(m)}(\tilde{t})=V_{h, i}^{(m)} \exp \left(\Gamma_{h}^{(m)} \tilde{t}\right)
$$

where $V_{h, i}^{(m)}$ denotes the initial volume of the gas in the isothermal expansion, $T_{s h}^{(m)}$ is given in Eq.(49), and the isothermal expansion rate of the $m$-th cycle $\Gamma_{h}^{(m)}$ is defined as

$$
\Gamma_{h}^{(m)} \equiv \frac{C_{h}\left(T_{h}^{[i]}-T_{b}\right)}{M N T_{s h}^{(m)} t_{h}^{(m)}} .
$$

The finial volume of the isothermal expansion follows as $V_{h, f}^{(m)}=V_{h, i}^{(m)} \exp \left(\Gamma_{h}^{(m)} t_{h}^{(m)}\right)$.

(B). For adiabatic expansion process of the $m$-th cycle, according to the adiabatic equation of ideal gas, i.e., $T_{s} V^{\xi-1}=$ const., one has

$$
T_{s h}^{(m)}\left[V_{h, f}^{(m)}\right]^{\xi-1}=T_{s c}^{(m)}\left[V_{c, i}^{(m)}\right]^{\xi-1},
$$

where $T_{s c}^{(m)}$ is the constant temperature of the gas during the isothermal compression, $V_{c, i}^{(m)}$ denotes the initial (final) volume of the gas in the isothermal compression (adiabatic expansion) process, and $\xi$ is the heat capacity ratio.

(C). In the finite-time isothermal compression process with the duration $t_{c}^{(m)}$, similar to process $\mathbf{A}$, the exothermic heat flow $q_{c}^{(m)}$ of the $m$-th cycle reads

$$
q_{c}^{(m)}=\kappa_{c}\left[T_{s c}^{(m)}-T_{c}^{(m)}\right]
$$

Note that the heat release from the working substance to the cold reservoir is exactly the total heat absorbed to the cold reservoir in $m$-th cycle, namely, $q_{c}^{(m)} t_{c}^{(m)}=\dot{Q}_{c} \tau_{c}$. Then, with the help of Eq. (45), the temperature of the gas $T_{s c}^{(m)}$ is obtained as

$$
T_{s c}^{(m)}=T_{c}^{(m)}+T_{c}^{(m)} \frac{C_{h}\left[T_{h}^{[i]}-T_{b}\right]}{M \sqrt{L_{22}} \kappa_{c} t_{c}^{(m)}}\left(\frac{C_{h}\left[T_{h}^{[i]}-T_{b}\right]}{\sqrt{L_{22}} \tau}+\frac{\sqrt{L_{22}}}{T_{h}^{(m)}}\right)
$$


In this process, the energy conservation relation follows as

$$
0=C_{s} d T_{s c}^{(m)}=-q_{c}^{(m)} d t-\frac{N T_{s c}^{(m)}}{V_{c}^{(m)}} d V_{c}^{(m)}
$$

which determines the optimal protocol of the isothermal compression as $V_{c}^{(m)}\left(t^{\prime}\right)=V_{c, i}^{(m)} \exp \left(-\Gamma_{c}^{(m)} t^{\prime}\right)$. Here, the isothermal compression rate of the $m$-th cycle $\Gamma_{c}^{(m)}$ is defined as

$$
\Gamma_{c}^{(m)} \equiv \frac{T_{c}^{(m)}}{T_{s c}^{(m)}} \frac{C_{h}\left[T_{h}^{[i]}-T_{b}\right]}{M N \sqrt{L_{22}} t_{c}^{(m)}}\left(\frac{C_{h}\left[T_{h}^{[i]}-T_{b}\right]}{\sqrt{L_{22}} \tau}+\frac{\sqrt{L_{22}}}{T_{h}^{(m)}}\right)
$$

$t^{\prime} \equiv t-\left[(m-1) \tau_{c}+t_{h}^{(m)}\right]\left(t^{\prime} \in\left[0, t_{c}^{(m)}\right]\right)$ is introduced, and $T_{s c}^{(m)}$ is given in Eq.(56). The finial volume of the isothermal compression is calculated as

$$
V_{c, f}^{(m)}=V_{c, i}^{(m)} \exp \left(-\Gamma_{c}^{(m)} t_{c}^{(m)}\right)=V_{h, i}^{(m)}\left[\frac{T_{s h}^{(m)}}{T_{s c}^{(m)}}\right]^{\frac{1}{\xi-1}} \exp \left(\Gamma_{h}^{(m)} t_{h}^{(m)}-\Gamma_{c}^{(m)} t_{c}^{(m)}\right)
$$

(D). For adiabatic expansion process of the $m$-th cycle, similar to process (B), we have $T_{s c}^{(m)}\left[V_{c, f}^{(m)}\right]^{\xi-1}=$ $T_{s h}^{(m)}\left[V_{h, i}^{(m)}\right]^{\xi-1}$. In order to make this adiabatic equation consistent with Eq. (59), the relation $\Gamma_{h}^{(m)} t_{h}^{(m)}=\Gamma_{c}^{(m)} t_{c}^{(m)}$ need to be satisfied. Combining with Eqs. (53) and (58), this relation is explicitly obtained as

$$
\frac{1}{\kappa_{h} t_{h}^{(m)}}+\frac{1}{\kappa_{c} t_{c}^{(m)}}=\frac{M\left[T_{h}^{(m)}\right]^{2}}{C_{h}\left[T_{h}^{[i]}-T_{b}\right] T_{h}^{(m)}+L_{22} \tau}
$$

which, together with $t_{h}^{(m)}+t_{c}^{(m)}=\tau_{c}$, determines the operation duration of the two finite-time isothermal processes in each cycle.

The protocols obtained in the above four processes complete the full operation scheme of the $m$-th cycle. In practice, we fix the initial value $V_{h, i}^{(m)}$ of the volume of the isothermal expansion in each cycle, namely, $V_{h, i}^{(m)}=V_{h, i}$. The finial volume $V_{h, f}^{(m)}$ of the isothermal expansion, the initial volume $V_{c, i}^{(m)}$ of the isothermal compression, and the final volume $V_{c, i}^{(m)}$ of the isothermal compression vary with $m$, and can be explicitly determined with the full operation protocols.

[1] M. J. Ondrechen, B. Andresen, M. Mozurkewich, and R. S. Berry, Am. J. Phys. 49, 681 (1981).

[2] Y. Izumida and K. Okuda, Phys. Rev. Lett. 112, 180603 (2014).

[3] Y.-H. Ma, Entropy 22, 1002 (2020).

[4] Y. Wang, Phys. Rev. E 90, 062140 (2014).

[5] Y.-H. Ma, D. Xu, H. Dong, and C.-P. Sun, Phys. Rev. E 98, 022133 (2018).

[6] Y.-H. Ma, R.-X. Zhai, J. Chen, H. Dong, and C. P. Sun, Phys. Rev. Lett. 125, 210601 (2020).

[7] M. Esposito, R. Kawai, K. Lindenberg, and C. V. den Broeck, Phys. Rev. Lett. 105, 150603 (2010).

[8] F. L. Curzon and B. Ahlborn, Am. J. Phys. 43, 22 (1975). 